\title{
Cellular uptake and anticancer effects of mucoadhesive curcumin-containing chitosan nanoparticles
}

\begin{abstract}
Curcumin, which is derived from turmeric has gained much attention in recent years for its anticancer activities against various cancers. However, due to its poor absorption, rapid metabolism and elimination, curcumin has a very low oral bioavailability. Therefore, we have formulated mucoadhesive nanoparticles to deliver curcumin to the colon, such that prolonged contact between the nanoparticles and the colon leads to a sustained level of curcumin in the colon, improving the anticancer effect of curcumin on colorectal cancer. The current work entails the ex vivo mucoadhesion study of the formulated nanoparticles and the in vitro effect of mucoadhesive interaction between the nanoparticles and colorectal cancer cells. The ex vivo study showed that curcumin-containing chitosan nanoparticles (CUR-CS-NP) have improved mucoadhesion compared to unloaded chitosan nanoparticles (CS-NP), suggesting that curcumin partly contributes to the mucoadhesion process. This may lead to an enhanced anticancer effect of curcumin when formulated in CUR-CS-NP. Our results show that CURCS-NP are taken up to a greater extent by colorectal cancer cells, compared to free curcumin. The prolonged contact offered by the mucoadhesion of CUR-CS-NP onto the cells resulted in a greater reduction in percentage cell viability as well as a lower IC50, indicating a potential improved treatment outcome. The formulation and free curcumin appeared to induce cell apoptosis in colorectal cancer cells, by arresting the cell cycle at G2/M phase. The superior anticancer effects exerted by CUR-CS-NP indicated that this could be a potential treatment for colorectal cancer.
\end{abstract}

Keyword: Curcumin; Nanoparticles; Mucoadhesion; Colorectal cancer; Cellular uptake 